\title{
Pratiques
}

Linguistique, littérature, didactique

179-180 | 2018

Poésie et langue : aspects théoriques et didactiques

\section{Un poème entre réfractions littéraires et scolaires}

A poem between literary and school refractions

\section{Yann Vuillet}

\section{(2) OpenEdition}

\section{Journals}

Édition électronique

URL : http://journals.openedition.org/pratiques/5170

DOI : 10.4000/pratiques. 5170

ISSN : 2425-2042

\section{Éditeur}

Centre de recherche sur les médiations (CREM)

\section{Référence électronique}

Yann Vuillet, « Un poème entre réfractions littéraires et scolaires », Pratiques [En ligne], 179-180 | 2018, mis en ligne le 31 décembre 2018, consulté le 21 avril 2019. URL : http://journals.openedition.org/ pratiques/5170 ; DOI : 10.4000/pratiques.5170

Ce document a été généré automatiquement le 21 avril 2019

(c) Tous droits réservés 


\title{
Un poème entre réfractions littéraires et scolaires
}

\author{
A poem between literary and school refractions
}

\section{Yann Vuillet}
Comprendre n'est jamais que traduire, c'est-à-dire donner l'équivalent d'un texte mais non point sa raison. Il n'y a rien derrière la page écrite, pas de double fond qui nécessite le travail d'une intelligence autre, celle de l'explicateur; pas de langue du maître, de langue de la langue dont les mots et les phrases aient le pouvoir de dire la raison des mots et des phrases d'un texte.
J. Rancière (2017, p. 20)

1 Cet article, qui reprend des analyses développées dans une section de ma thèse de doctorat (Vuillet, 2017), propose d'approcher la Vénus Anadyomène ${ }^{1}$ d'A. Rimbaud (2009) dans une perspective de recherche didactique. En empruntant une formule ainsi que quelque chose d'une manière de penser au maitre ignorant de J. Rancière, je précise d'emblée que je ne tenterai pas de «dire la raison des mots et des phrases " (Rancière, 2017, p. 20) qui composent ce sonnet. Plutôt que de produire un discours qui voudrait l'expliquer, je comparerai ce qui a pu être dit et fait de la V.A. au sein de contextes sociohistoriques distincts: ceux d'études littéraires académiques - à travers des commentaires que l'on doit à J. Plessen (1967), à M. Rifaterre (1979) et à S. Murphy (1990) - et celui d'une séquence d'enseignement réalisée en 2015 dans une classe de $10^{\mathrm{e}}$ Harmos du secondaire I genevois ${ }^{2}$.

2 La démarche comparatiste annoncée implique quelques présupposés qu'il convient de préciser. À la suite de propositions formulées notamment par D. Maingueneau (2004, 2006) au sujet du discours littéraire, je considère qu'il ne saurait y avoir de rapport de stricte externalité entre un texte (quel qu'il soit) et ses divers contextes de production, de lecture, de commentaire ou de mise en discussion. En m'inspirant simultanément de thèses développées par J.-P.Bronckart, j'aimerais également montrer que les 
connaissances de toutes sortes développées au sujet ou à partir d'un poème ne reflètent pas directement ce dernier mais qu'elles réfractent, en leur sein, les "propriétés historiques, sociales et culturelles des cadres au sein desquels elles ont été élaborées » (Bronckart, 2008, p. 862).

3 S'agissant du corpus constitué, les activités d'experts académiques ainsi que les activités scolaires effectuées à l'endroit de la V.A. seront donc approchées comme dépendant, respectivement, de leurs propres conditions de réalisation. Une fois mises en évidence et comparées quelques-unes des propriétés de ces ensembles d'activités, la démarche adoptée me conduira finalement à argumenter en faveur de la reconnaissance d'une légitimité propre, bien distincte de celle des études littéraires académiques, au travail scolaire pouvant être effectué à l'endroit de textes réputés poétiques.

\section{La Vénus Anadyomène d'A. Rimbaud}

Dans cet article, la V.A. est le point commun minimal reliant les activités de la critique experte et les activités scolaires qui seront analysées. Ce sonnet est utilisé comme un réactif (Ronveaux \& Schneuwly, 2018) permettant de contraster les propriétés des deux contextes approchés. En voici une présentation succincte, voulue relativement neutre.

5 A. Rimbaud compose la V.A. en 1870, à l'âge de dix-sept ans. Ce texte apparait dans les Cahiers de Douai (2009) et constitue le huitième texte du recueil.

Vénus Anadyomène

Comme d'un cercueil vert en fer blanc, une tête

De femme à cheveux bruns fortement pommadés

D'une vieille baignoire émerge, lente et bête,

Avec des déficits assez mal ravaudés ;

Puis le col gras et gris, les larges omoplates

Qui saillent; le dos court qui rentre et qui ressort ;

Puis les rondeurs des reins semblent prendre l'essor ;

La graisse sous la peau paraît en feuilles plates ;

L'échine est un peu rouge, et le tout sent un goût

Horrible étrangement ; on remarque surtout

Des singularités qu'il faut voir à la loupe...

Les reins portent deux mots gravés : Clara Venus ;

- Et tout ce corps remue et tend sa large croupe

Belle hideusement d'un ulcère à l'anus.

Il s'agit d'un sonnet qui s'empare du thème antique de la naissance de Vénus/Aphrodite d'une manière qui pourrait être qualifiée d'originale, de corporelle, de sexuelle. À elles seules cependant, ces quelques considérations illustrent qu'il ne saurait y avoir de description absolument neutre de ce poème. Produire le commentaire d'un poème implique inévitablement un énonciateur, un contexte d'énonciation, un ou plusieurs destinataires, et dépend de l'activation d'un cadre herméneutique (au minimum) : la section suivante va l'illustrer à travers trois commentaires de la V.A. tirés d'études littéraires.

\section{Trois commentaires experts de la Vénus Anadyomène}

7 Je propose maintenant de rendre visible ce que réfractent trois commentaires du sonnet, et dont sont auteurs autant d'experts académiques. Les discours retenus pour l'occasion, 
produits par J. Plessen (1967), M. Riffaterre (1979) et S. Murphy (1990) sur une grande vingtaine d'années, divergent quant aux cadres herméneutiques qu'ils activent. D'un point de vue didactique, il est notable que ces cadres - respectivement qualifiables de normatif, de formel, et d'historico-culturel - correspondent à autant de périodes identifiées dans l'évolution, au XX ${ }^{\mathrm{e}}$ siècle, de l'enseignement de «la » littérature (Legros \& Canvat, 1997 ; Dufays, Gemenne \& Ledur, 2005).

Malgré leur diversité et leur espacement temporel relatif, les trois approches de ces experts sont traversées d'un type de positionnement du commentateur vis-à-vis du poème qui peut être reconnu comme identique. Ce positionnement oriente la construction par chacun, à travers son propre discours mais à la manière des autres, d'une identité et d'une légitimité d'expert littéraire. Il correspond en cela au fonctionnement de l'herméneutique littéraire, tel que décrit par l'analyste du discours D. Maingueneau (2006, p. 63) :

L'enseignant ou le critique qui font une stimulante interprétation de Molière montrent pragmatiquement à la fois que l'interprète est compétent et que le texte est d'une grande richesse, et, au-delà, que la littérature est bien un discours constituant, associé à un cadre herméneutique. [...] Tout commentaire exemplaire est bien autre chose qu'une activité réussie: il conforte l'institution et la communauté qu'elle implique, provoquant une double reconnaissance, celle de l'interprète, celle du cadre herméneutique lui-même.

Dans une perspective didactique, une analyse des commentaires de J.Plessen, de M. Riffaterre et de S.Murphy sous l'angle proposé par D. Maingueneau a ceci d'intéressant qu'elle peut participer concrètement, je crois, à l'historicisation et donc à la dénaturalisation (Daunay, 2007) de rapports contextuels à un texte réputé littéraire (Vuillet \& Gabathuler, 2017 ; Védrines \& Gabathuler, 2018).

\section{J. Plessen : propositions normatives et intimistes}

La première "lecture" que je retiens de la V.A. prend la forme d'une collection d'allusions produites par J. Plessen (1921-2007), spécialiste d'A. Rimbaud ayant occupé la fonction de professeur de littérature française à l'université d'Utrecht de 1972 jusqu'à sa retraite en 1986. Dans sa thèse de 1967, J. Plessen consacre les quelques passages suivants à la V.A. :

- "S'attaquer à ce qu'on aime est, bien sûr, une forme de masochisme, qu'on peut également voir à l'œuvre dans la misogynie rancunière que Rimbaud exhale, par exemple, dans Vénus Anadyomène, où, en salissant l'image de la femme, il se punit d'avoir cru à la rédemption par l'amour de la déesse [...]. » (ibid., p. 37)

- « [...] la charge rancunière qu'est l'ironique Vénus Anadyomène [...]. » (ibid., p. 121)

- "Le mystère du “Chanaan féminin" l'a évidemment intrigué, mais l'horreur qu'il lui inspire semble avoir prévalu. Vénus Anadyomène [...] est une tentative pour conjurer cette terreur 
par le ricanement. [...] la sodomie pratiquée par Rimbaud ne serait-elle pas une déviation de l'instinct sexuel dictée par la peur devant le sexe féminin ?» (ibid., p. 158)

11 Ces extraits, soit tous ceux qui se réfèrent explicitement à la V.A. dans l'ouvrage, font apparaitre à la troisième personne du singulier des termes à forte valeur axiologique. Ces derniers renvoient tour à tour à des registres :

- psychologique ou psychanalytique («misogynie rancunière que Rimbaud exhale »; "charge rancunière »; « l'horreur qu'il [le sexe féminin] lui inspire »; « déviation de l'instinct sexuel dictée par la peur devant le sexe féminin »),

- sacré (« il se punit d'avoir cru à la rédemption par l'amour de la déesse »),

- ou encore biographique ( le mystère du "Chanaan féminin” l'a évidemment intrigué »; «la sodomie pratiquée par Rimbaud »).

Les extraits en question donnent à voir que le poème de la V.A. a pu être saisi en 1967 par un expert rimbaldien dans un cadre herméneutique employant parfois le texte comme un moyen de « remonter » à des éléments psychiques de son auteur, ou employant d'autres fois la vie ou le psychisme de cet auteur comme un moyen d'expliquer ce qui se joue dans ses textes. Dans les deux cas, des touches normatives et intimistes parsèment les propositions de J. Plessen. Normatives, parce que nombre de termes employés par l'expert sont fortement connotés du point de vue moral. Intimistes, parce que ces propositions touchent à la sphère privée du poète, mettant en évidence que le spécialiste revendique, certes implicitement, une forme de proximité avec un auteur au sujet duquel il écrira pendant toute sa vie.

\section{Riffaterre : une « création toute formelle »}

Le second commentaire de la V.A. que je retiens est le fait de M. Riffaterre (1924-2006), théoricien de la littérature ayant marqué de ses travaux la critique littéraire du $\mathrm{XX}^{\mathrm{e}}$ siècle. Lexicologue, sémioticien, structuraliste, théoricien de l'intertextualité, les étiquettes qui peuvent le qualifier sont nombreuses et rendent compte d'une activité critique et théorique foisonnante. M. Riffaterre a obtenu sa thèse en 1955 à Columbia University, où il mène sa carrière académique depuis 1953 jusqu'à la retraite (il est nommé « University professor » en 1982, puis professeur émérite en 2004).

Dans son ouvrage La production du texte, M. Riffaterre (1979) consacre quelques pages du chapitre « Pour une approche formelle de l'histoire littéraire » à la V.A. Ce titre annonce un engagement théorique explicite : le "pour» laisse entendre que la réflexion à venir aura une portée argumentative. Pour cet expert, le poème d'A. Rimbaud consiste en une «laus Veneris inverse, construite a contrario.» (ibid., p. 94). M. Riffaterre développe son argumentaire comme suit :

Bref, il s'agit du contreblason. Car Rimbaud ne fait pas la satire de son époque: certains critiques ont cru que le sonnet faisait pendant à un autre poème que Rimbaud écrivit à la gloire de la beauté grecque, qu'il moquait notre âge de fer, miroir déformant du Beau classique. Or le contreblason ne parodie pas les genres encomiastiques, comme on le dit un peu vite; il n'est ni polémique morale ni outil de controverse esthétique. Le sonnet de Rimbaud n'est pas écrit contre, mais «à l'envers ». Il n'est ni satire ni parodie et il n'oppose pas la modernité au classicisme. C'est un exercice de transformation, c'est un cas exemplaire de conversion. [...] Il appartient déjà à une littérature fondée tout entière sur le potentiel des associations lexicales, sur une création toute formelle, où les mots sont arrangés visiblement par référence à un modèle verbal, et non par référence au réel. Écriture 
au miroir, qui reflète un autre texte. Le physicien déduit l'antimatière des propriétés connues de la matière. Rimbaud déduit une antireprésentation. (ibid., p. 96-97)

15 Dans le cadre herméneutique déployé, l'analyse produite au sujet de la V.A. cherche non seulement à l'expliquer, mais aussi à confirmer par son acuité que l'approche formelle de l'histoire littéraire fonctionne efficacement. Comme chez J. Plessen, l'énonciateur n'a pas besoin d'apparaitre directement dans l'extrait pour que sa stature soit assumée et son expertise démontrée : ses nombreux énoncés performatifs ont force d'autorité. L'expert non d'A. Rimbaud comme c'était le cas avec J. Plessen, mais plutôt d'une approche théorique dont la diffusion est visée - occupe un point de vue voulu neutre et surplombant sur les phénomènes qu'il décrit. Ce point de vue ne va pas sans rappeler le paradigme des sciences exactes.

L'approche formelle implique quelques contraintes qui se retrouvent dans la pratique discursive de l'expert littéraire. Il convoque des savoirs à prétention objective: par exemple sur les genres littéraires (« contreblason»; « genres encomiastiques »), sur les phénomènes intertextuels (« satire »; parodie »; « exercice de transformation »), ou sur des fonctionnements lexicaux («associations lexicales»; «référence verbale »). Il nie parallèlement la pertinence d'autres entrées possibles dans ce poème, ainsi celle des discours des experts l'ayant précédé («certains critiques ont cru que [...]»; «le contreblason ne parodie pas [...] comme on le dit un peu vite»). Il réfute enfin, globalement, que le poème puisse avoir un référent réel ou social (« ni polémique morale, ni outil de controverse esthétique "; « littérature fondée tout entière sur le potentiel des associations lexicales [...], où les mots sont arrangés visiblement par référence à un modèle verbal, et non par référence au réel »).

17 L'acte du commentateur se conçoit, ici, dans un cadre où il s'agit de prétendre à une distance objective et scientifique face au texte afin de ne pas lui faire dire autre chose que ce qu'il voudrait dire. Pour M. Riffaterre, cette distance est garante du fait que le texte imposera son effet à la longue - et ce, quelles que soient les « vicissitudes historiques » ( ibid., p. 98). Paradoxalement, l'approche formelle de l'histoire littéraire voulue par M. Riffaterre a pour effet de réduire la variable historique au statut de ce qui peut retarder ou suspendre le déclenchement, par le texte même, de la lecture qu'il appelle. Couper l'énoncé littéraire de ses dimensions contextuelles est le prix à payer pour que l'expert puisse prétendre être fidèle à son objet. Mais encore le commentateur se coupe-til lui-même, du même mouvement, de son propre lieu social d'énonciation - l'étude littéraire académique - pour assurer sa position de surplomb théorique. Alors que le paradigme de ce cadre herméneutique est celui des propriétés du monde objectif, le monologisme de la méthode formelle ainsi décrite devient manifeste: prétendument située au-delà des «vicissitudes » historiques et sociales, elle ne se discute littéralement pas.

\section{S. Murphy : la « nature de rapports conflictuels entre auteur et lecteur»}

18 Le troisième commentaire de la V.A. que j'évoquerai est développé par S. Murphy. Né en 1956, et professeur de littérature française à l'université Rennes 2 Haute-Bretagne, S. Murphy est un spécialiste reconnu d'A. Rimbaud, et plus largement de la poésie française du XIX ${ }^{e}$ siècle. L'approche privilégiée par S. Murphy tend à comprendre les 
contextes sociohistoriques (de production, mais aussi dans une certaine mesure de réception) pour aborder les textes. De même, les problématiques esthétiques ne sont pas mises à distance des enjeux éthiques dans les réflexions de cet expert.

En 1990, dans le premier ouvrage que S. Murphy consacre à A. Rimbaud, le spécialiste développe une étude particulièrement exhaustive de la V.A. Comme chez J. Plessen et M. Riffaterre, elle déploie des ressources rhétoriques pour légitimer circulairement le texte, son auteur, son commentateur et le cadre herméneutique que ce dernier déploie. Pour sa part, le cadre herméneutique de S. Murphy intègre assez profondément dans son système ce que d'autres experts ont dit, ou fait dire au poème. Tout en contredisant ces discours précédents, S. Murphy s'appuie sur eux pour mettre en évidence ce qu'A. Rimbaud déclencherait avec la V.A. :

Vénus Anadyomène est incontestablement l'un des poèmes les plus provocateurs de la langue française. Il pose et expose crûment la nature de rapports conflictuels entre auteur et lecteur. Rimbaud sollicite victorieusement des réactions négatives : dégoût, colère et indignation. Il refuse tout compromis. D'où, de la part de ses commentateurs, l'élaboration de stratégies tendant à neutraliser son message. Que l'on ait adopté une approche formaliste, mettant entre parenthèses son "contenu", ou que l'on ait au contraire, par un biographisme simpliste, fait du texte une pure donnée psychopathologique, on a presque toujours fini par esquiver le détail sémantique du sonnet, comme si, sur le plan lexical par exemple, tout était transparent. Il n'en est rien. Et l'on peut penser que cet escamotage n'a pas toujours été accidentel et inconscient. De la sorte, on est parvenu non seulement à occulter les objectifs de Rimbaud, mais très souvent aussi à les inverser. (Murphy, 1990, p. 190)

20 Une différence intéressante entre l'approche de S. Murphy et celles de ses prédécesseurs tient à ce qu'il thématise explicitement les émotions ("dégoût ", "colère ", «indignation») que le poème serait supposé déclencher chez ses lecteurs. Plus exactement, ce ne sont pas les réactions émotionnelles de lecteurs lambda que convoque le critique, mais celles des experts de la V.A. qui l'ont précédé dans l'exercice. Ces émotions - sans que l'on sache d'ailleurs par quelle méthode S. Murphy parvient à les nommer - ont un rôle de pivot argumentatif dans l'extrait. Le critique les voit à l'origine du «fait» que ses prédécesseurs auraient cherché à "neutraliser» ce que serait le "message » du poème. Elles auront donc eu un effet néfaste sur les commentaires précédents, quelles que soient leurs perspectives herméneutiques respectives.

21 Les exemples donnés par S. Murphy peuvent être mis en lien avec leurs auteurs. L'« approche formaliste, mettant entre parenthèses son "contenu" » renvoie sans mystère à $\mathrm{M}$. Riffaterre, qui se trouve plus loin nommément pris à partie ${ }^{3}$. Lorsque se trouve évoqué un "biographisme simpliste» ayant «fait du texte une pure donnée psychopathologique ", il fait peu de doute que ce soient les allusions de J. Plessen qui se trouvent visées. S. Murphy présente ainsi ces discours d'experts, qu'il considère erronés, comme représentatifs du fait que «Rimbaud sollicite victorieusement des réactions négatives ». En d'autres termes, le critique exploite les commentaires littéraires de ses prédécesseurs pour reconstruire une intentionnalité auctoriale et pour conforter la supériorité du poète sur les experts. Tout en étendant aux commentaires de M. Riffaterre une assignation d'insuffisance que ce dernier avait lui-même précédemment dirigée vers les analyses de J. Plessen, S. Murphy prend sa place dans la chaine des exégèses expertes : pour ce faire, il doit bien montrer que le texte est si exigeant qu'il lui faut des commentateurs aguerris, mais encore, que ceux qui se sont essayés à l'explication avant lui n'y sont pas parvenus. S. Murphy contredit donc, en toute logique, la pertinence des 
cadres herméneutiques déployés par d'autres, et assoit du même geste la supériorité de celui qu'il développe. Dans cette perspective, S. Murphy légitime non seulement A. Rimbaud dans son statut de figure surplombante de la poésie du XIX ${ }^{\mathrm{e}}$ siècle, mais il se légitime lui-même dans sa position de commentateur privilégié.

\section{Réfractions littéraires du poème}

Le constat général suivant peut être tiré des analyses effectuées: bien que J. Plessen, M. Riffaterre et S. Murphy convoquent des cadres herméneutiques contrastés pour aborder la V.A., et alors même qu'ils la dotent par ces intermédiaires de significations historiques, esthétiques et sociales passablement différentes, des principes constants traversent leurs commentaires respectifs. Très proches du fonctionnement des discours constituants décrits par D. Maingueneau $(2004,2006)$ les commentaires analysés tendent à légitimer circulairement, non sans quelques différences dans les focales ou les procédés :

- La qualité rare, la complexité et l'intérêt du sonnet de la V.A. ;

- la position de surplomb occupée par l'auteur (A. Rimbaud) dans l'histoire des productions réputées littéraires ;

- la maitrise par les commentateurs de cadres herméneutiques rendant possible la formulation de commentaires ;

- la position de surplomb critique et de proximité avec le poète ou son œuvre pour les commentateurs.

$\mathrm{Au}$ sortir de ces analyses, ce qui a pu être fait de la V.A. dans des contextes de critique littéraire experte apparait assez clairement. En résumé, le poème peut être vu comme étant pris dans un ensemble d'activités d'analyses et de production de discours, activités qui réalisent et actualisent des modes de productions de connaissances ainsi que des formes, régulières, de légitimation littéraire académique. Après avoir décrit certains aspects desdits modes et formes, je vais tenter maintenant de rendre visible ce que peuvent réfracter, de leur côté, des activités scolaires ayant été réalisées à partir, ou autour du même poème.

\section{Une séquence d'enseignement réalisée à partir de la Vénus Anadyomène}

Avant d'analyser les activités scolaires retenues pour cet article, je précise que ces dernières ne sont pas approchées dans le but de leur reconnaitre une quelconque valeur d'exemplarité. Il ne s'agit pas dans cette section de vouloir prescrire des manières de faire, de fournir un modèle à suivre. Il s'agit de décrire et de rendre intelligible, dans une perspective de recherche didactique, une unique transformation avérée de la V.A. en objet d'enseignement.

Afin de reconstruire le déploiement de l'objet d'enseignement dans la séquence organisée autour de la V.A., je me focaliserai sur le geste professionnel de mise en place d'un dispositif didactique (au sens de Schneuwly, 2009, p. 37-38). Je m'appuierai pour ce faire sur la macrostructure de la séquence ${ }^{4}$ (Dolz, Jacquin \& Schneuwly, 2006 ; Aeby Daghé et al., 2009). Cette macrostructure, présentée exhaustivement dans ma thèse de doctorat (Vuillet, 2017), est réduite dans le présent article à une vue d'ensemble. Elle a été réalisée à partir 
des transcriptions des interactions orales produites au cours des 11 séances réalisées (soit approximativement 8 heures d'enseignement).

\section{Les dix modules de la séquence : une orientation esthétique, socio- culturelle et historique}

La séquence approchée développe 10 modules distincts. Le tableau 1, ci-dessous, propose un intitulé pour chaque module et décrit synthétiquement ce qui s'y passe, sans entrer dans la variété des tâches et sous-tâches. Les modules sont numérotés par ordre d'apparition dans le déroulé de la séquence 5 .

Tableau 1. Vue d'ensemble des 10 modules de la séquence d'enseignement

\begin{tabular}{|c|c|}
\hline Intitulés des modules & Description synthétique \\
\hline $\begin{array}{l}\text { 1. Problématisation poésie } \\
\text { et beauté }\end{array}$ & $\begin{array}{l}\text { Les élèves explicitent et mettent en discussion leurs conceptions de } \\
\text { la poésie, de la beauté et de leurs rapports possibles. }\end{array}$ \\
\hline $\begin{array}{l}\text { 2. Discussion avec des } \\
\text { tableaux : représentations } \\
\text { classiques - thème, } \\
\text { naissance Vénus }\end{array}$ & $\begin{array}{l}\text { Les élèves discutent de représentations "classiques" du thème de } \\
\text { la naissance de Vénus/Aphrodite. Ils entrent en contact avec un } \\
\text { récit de la naissance d'Aphrodite adapté de la Théogonie d'Hésiode } \\
\text { (VIII }{ }^{\mathrm{e}} \text { siècle av. J.-C.), puis avec quatre interprétations picturales de } \\
\text { la naissance de Vénus par Botticelli (XV siècle); Titien (XVI } \\
\text { siècle); Ingres (XIX }{ }^{\mathrm{e}} \text { siècle); Cabanel (XIX siècle). }\end{array}$ \\
\hline $\begin{array}{l}\text { 3. Découverte de la V.A. et } \\
\text { de deux interprétations } \\
\text { musicales }\end{array}$ & $\begin{array}{l}\text { Les élèves lisent deux fois la V.A. individuellement, débattent quant } \\
\text { à savoir s'il s'agit ou non de poésie, puis discutent de deux } \\
\text { interprétations musicales contemporaines de la V.A. (une version } \\
\text { par le comédien J.-C. Dreyfus [2008]; une version par le groupe } \\
\text { punk Cheveu [2006]). }\end{array}$ \\
\hline $\begin{array}{l}\text { Questionnaire } \\
\text { appréciation justifiée }\end{array}$ & $\begin{array}{l}\text { Les élèves, par écrit et en justifiant leurs positions, expliquent ce } \\
\text { qu'ils apprécient et/ou n'apprécient pas du poème de la V.A., ou } \\
\text { expriment leur possible indifférence. }\end{array}$ \\
\hline $\begin{array}{l}\text { 5. Mise en scène et mise } \\
\text { en voix de la V.A. par } \\
\text { l'enseignant }\end{array}$ & $\begin{array}{l}\text { Les élèves assistent à une mise en scène de la V.A. dite par } \\
\text { l'enseignant. }\end{array}$ \\
\hline $\begin{array}{l}\text { 6. Approche syntaxique et } \\
\text { grammaticale du premier } \\
\text { quatrain }\end{array}$ & $\begin{array}{l}\text { Les élèves cherchent à reconstruire une syntaxe pouvant faciliter la } \\
\text { compréhension du premier quatrain. Ils procèdent à une analyse } \\
\text { grammaticale des constituants de la phrase. }\end{array}$ \\
\hline 7. Paraphrase V.A. & $\begin{array}{l}\text { Les élèves recherchent des synonymes ou des reformulations pour } \\
\text { les mots ou syntagmes du poème qu'ils ont préalablement } \\
\text { identifiés comme n'étant pas compris par eux. }\end{array}$ \\
\hline $\begin{array}{l}\text { 8. Discussion avec un } \\
\text { tableau : comparaison V.A. } \\
\text { et Botticelli }\end{array}$ & $\begin{array}{l}\text { Les élèves mettent en perspective et comparent la V.A. et la } \\
\text { représentation picturale que Botticelli a proposée de la naissance } \\
\text { de Vénus. }\end{array}$ \\
\hline
\end{tabular}




\begin{tabular}{|l|l|}
\hline $\begin{array}{l}\text { 9. Transposition V.A. sous } \\
\text { forme de calligramme }\end{array}$ & $\begin{array}{l}\text { Les élèvent produisent un calligramme individuel pour une strophe } \\
\text { du poème qui leur a été attribuée. }\end{array}$ \\
\hline $\begin{array}{l}\text { 10. Discussion avec un } \\
\text { tableau : effets esthétiques } \\
\text { de « re-créations " }\end{array}$ & $\begin{array}{l}\text { Les élèves discutent de l'effet esthétique (embellissement/ } \\
\text { enlaidissement) qui peut être réalisé ou recherché lors d'une « re- } \\
\text { création » à partir d'un thème. Ils discutent de la représentation } \\
\text { picturale de la V.A. par la peintre Ida (XXI siècle), et de leur propre } \\
\text { transposition d'une strophe de la V.A. en calligramme. }\end{array}$ \\
\hline
\end{tabular}

La synthèse descriptive des dix modules ci-dessus permet de relever que la séquence d'enseignement déployée autour de la V.A. aborde entre autres des «contenus » relatifs à :

- L'esthétique (réflexions autour de la "beauté »; appréciation du sonnet et des différentes productions d'ordre culturel abordées durant la séquence) ;

- la poésie et les genres poétiques (réflexions autour de la "poésie»; production d'un calligramme à partir du sonnet de la V.A.) ;

- la mythologie (thème de la naissance de Vénus/Aphrodite);

- l'histoire de l'art (interprétations picturales du thème de la naissance de Vénus; interprétations musicales et picturales du sonnet de la V.A.) ;

- certains fonctionnements langagiers à l'œuvre dans le sonnet de la V.A. (syntaxe; analyse grammaticale ; lexique ; production d'une paraphrase collective de l'ensemble du sonnet).

- La production d'évaluations individuelles et la mise en débat collectif de ces dernières.

Pour qualifier globalement l'orientation de la séquence, sur la base de ce qui précède, il pourrait être dit qu'elle relève d'une perspective esthétique, socioculturelle et historique. Cette orientation se manifeste à travers un ensemble de phénomènes impossibles à analyser exhaustivement dans le cadre de cet article ${ }^{6}$. Parmi ces phénomènes, je retiendrai ici la grande variété d'interprétations du thème de la naissance de Vénus/ Aphrodite convoquées ou produites dans les situations scolaires approchées: cette particularité permet en effet de rendre visibles quelques différences entre le traitement de la V.A. dans les contextes comparés.

D'Hésiode au groupe de punk Cheveu, il est ainsi notable que les élèves entrent en contact avec pas moins de neuf versions différentes de la naissance de Vénus/Aphrodite, qu'elles soient picturales, écrite ou musicales (modules $2 ; 3 ; 8$ ). À ces versions avérées en dehors de l'école s'ajoutent également d'autres interprétations scolaires. Les élèves assistent à trois mises en scène de la diction du poème par l'enseignant (module 5), avant de se voir eux-mêmes amenés à produire différentes paraphrases du poème (module 7). Enfin, la séquence se conclut peu après l'effectuation d'une transposition générique (au sens de Calame-Gippet \& Marcoin, 1999), à la fois individuelle et collective, de la V.A. sous forme de calligramme $\mathrm{e}^{7}$ (module 9). Au total, en comptabilisant indistinctement les productions d'artistes didactisées ainsi que les productions scolaires, plus d'une quinzaine de traitements différents du thème de la naissance de Vénus/Aphrodite peuvent être dénombrés. Il est donc possible d'ajouter que la séquence approche le sonnet de la V.A. non pas en tant qu'un texte dont il s'agirait de retrouver le(s) sens caché(s) - aucune activité de ce type n'apparait dans la séquence - mais plutôt en tant qu'une (ré)interprétation, parmi d'autres, du thème de la naissance de la déesse de la beauté. 


\section{Réfractions scolaires du poème}

30 Afin de rendre intelligibles certaines propriétés du contexte approché, telles que les réfractent les activités scolaires développées autour du poème, je propose de voir ces dernières à travers les composantes du système didactique ${ }^{8}$.

Du côté des rapports entre le texte et les contenus d'enseignement tout d'abord, le travail effectué autour de la V.A. peut être vu comme conditionnant l'apprentissage, par les élèves, d'aspects d'activités humaines par lesquelles sont investies des productions culturelles à valeur esthétique et sociale. Dans cette perspective, le sonnet n'est pas considéré comme un donné indépassable. Bien plutôt est-il employé comme un exemple possible de production poétique, pouvant d'ailleurs faire l'objet de transformations ${ }^{9}$. La V.A. endosse le statut d'un outil didactique qui n'est pas abordé en classe pour lui-même. Cet outil rend possibles les transformations de "modes de penser, de parler et d'agir » des élèves (Schneuwly, 2009, p. 30-31) vis-à-vis des contenus d'enseignement.

Du côté de l'enseignant et des élèves, il peut être relevé ensuite que le déploiement de l'objet d'enseignement suppose, dans la séquence analysée, que l'ensemble des acteurs soient légitimés à manipuler la V.A., à intervenir sur elle, à la « recréer $»^{10}$ : l'enseignant se saisit du texte pour créer des tâches originales, tout comme les élèves, à travers ces tâches, la transforment de diverses manières. Cette légitimation scolaire, qui conditionne la réalisation même des activités d'enseignement et d'apprentissage, s'écarte ainsi des modes de légitimation littéraire précédemment mis en évidence au sein des commentaires d'experts. Agir avec ce poème, agir sur lui dans le contexte de cette classe implique en effet de pouvoir travailler sans la contrainte directe de devoir faire mieux, de devoir mieux dire que les prédécesseurs (qu'ils soient artistes ou experts littéraires) ${ }^{11}$. Il en ressort que le contexte scolaire réoriente ou redéfinit non seulement le statut du texte, mais qu'il peut également autoriser celles et ceux qui interagissent avec lui à adopter, à son égard, un positionnement autre que celui qui prévaut au sein des études littéraires.

Du côté des dimensions institutionnelles, il convient finalement de relever que les tâches orientant la construction de l'objet d'enseignement sont également en rapport avec ce que l'on pourra considérer comme la configuration disciplinaire (Reuter \& Lahanier-Reuter, 2007 ; Laurent, 2011) du français en Suisse romande. Ce qui permet de l'avancer, c'est que l'ensemble des six finalités de l'enseignement du français orientant le Plan d'Études Romand (comprendre et produire des textes oraux et écrits; travailler sur le fonctionnement de la langue; construire des références culturelles) se trouve en toile de fond de la séquence. Autrement dit, la logique de construction de l'objet d'enseignement dénote à la fois l'attribution d'un statut d'outil didactique au poème, la réalisation d'un processus de légitimation de l'enseignant et des élèves à agir avec et sur le texte, ainsi qu'un mode d'articulation de sous-disciplines constitutives du «français » comme discipline d'enseignement.

\section{Conclusion}

Les traitements avérés de la V.A. retenus dans cet article n'épuisent aucunement ce qui pourrait être dit et fait de ce poème. Il ne s'agissait d'ailleurs pas d'opposer telle activité à telle autre pour proclamer l'exemplarité de l'une d'elles : j'ai avant tout tenté de rendre intelligible le fait que toutes les connaissances prenant pour objet un même texte 
poétique ${ }^{12}$ entretiennent des rapports importants avec les propriétés historiques, sociales et culturelles de leurs contextes respectifs de production et de transmission. Je pense ainsi avoir illustré que le phénomène de réfraction (Bronckart, 2008) concerne bel et bien l'ensemble des activités approchées, qu'elles relèvent d'études littéraires ou d'une séquence d'enseignement. Ceci étant, en conclusion, j'aimerais insister sur quelques différences qui séparent le travail de l'enseignant de celui qu'effectuent les experts des études littéraires lorsqu'ils commentent des textes. Ces différences me semblent pouvoir éclairer de façon complémentaire les écarts de traitement de la V.A. qui sont apparus à travers les analyses effectuées.

Du point de vue des fonctions sociales des dépositaires d'une forme d'autorité textuelle que sont les experts littéraires académiques et les enseignants, il peut être remarqué que si les uns comme les autres ont en charge un travail avec des textes, les premiers ne s'intéressent professionnellement qu'à ceux qui sont réputés porteurs d'une valeur littéraire. Il revient par contre aux enseignants de se saisir, potentiellement, de l'ensemble des genres de textes retenus par le système scolaire pour faire l'objet d'enseignements. Cette relation différente aux textes entraine notamment que l'autorité des enseignants est moins directement tributaire de l'attribution de qualités exceptionnelles à un corpus donné - «la » littérature ; «la » poésie - que ce n'est le cas pour les experts littéraires académiques. Si ces derniers doivent sans cesse, dans un environnement particulièrement compétitif, donner les preuves de leur propre légitimité à commenter ces textes choisis (desquels ils doivent simultanément alimenter la réputation de grandeur), ce n'est pas nécessairement le cas pour les enseignants. Le travail de ces derniers consiste plus largement à s'adresser à des élèves en fonction de finalités scolaires, décidées politiquement, confiées institutionnellement, et qui contraignent explicitement leurs activités. Pour mener à bien les tâches qui leur échoient, les enseignants déploient ainsi des formes d'expertise qui ne sont ni supérieures ni inférieures à celles développées dans les études littéraires, mais qui relèvent simplement et nécessairement, pour une bonne partie, d'un autre registre.

Reconnaitre cet état de fait ne revient en aucune manière à contester la pertinence des rapports aux textes développés dans les études littéraires, ni même à proscrire l'abord, en classe, de rapports de ce type. Il s'agit plutôt, par cet intermédiaire, de rechercher des moyens théoriques susceptibles d'aider à observer et à comprendre ce qui se joue, d'un point de vue didactique, lorsque des professionnels prennent en charge la transmission d'un ensemble de savoirs disciplinaires vers les publics scolaires. La recherche de ces moyens de mise en intelligibilité importe d'autant plus que les textes poétiques, ou plus largement réputés littéraires, activent des processus de (dé-)valorisation sociale et mettent en place des effets de reconnaissance ou de distinction dont la justesse ne peut, je crois, être tranchée sur un plan strictement épistémologique ${ }^{13}$.

37 À l'école, comme c'est le cas de tout texte, la transformation nécessaire et inévitable des poèmes en outils didactiques plaide en somme pour reconnaitre une forme de légitimité propre aux activités scolaires ainsi qu'à leurs fonctionnements. Dans le cas de la poésie, cette légitimité appelle à ne pas considérer uniquement ce qui se passe en classe comme une transposition plus ou moins distante ou transformative de pratiques de commentaires académiques mais aussi, sinon surtout, comme des constructions profondément originales : des élaborations didactiques (Halté, 1992). 


\section{BIBLIOGRAPHIE}

AEBY DAGHÉ, S. et al. (2009). « Une analyse multifocale ». In : Schneuwly, B. \& Dolz, J. (éds), Des

objets enseignés en classe de français. Rennes : Presses universitaires de Rennes, p. 101-114.

BRONCKART, J.-P. (2008). « Discussion de quelques concepts pour une approche praxéologique du

langage ». In : Actes du $1^{\text {er }}$ Congrès mondial de linguistique française. p. 861-867.

CALAME-GIPPET, F. \& MARCoIN, D. (1999). « Des dispositifs didactiques et pédagogiques pour construire la lecture littéraire à l'école ». Repères 19, p. 61-101. En ligne : https://www.persee.fr/ doc/reper_1157-1330_1999_num_19_1_2291.

DAUNAY, B. (2007). «État des recherches en didactique de la littérature : note de synthèse ». Revue française de pédagogie 159, p. 139-189. En ligne : https://journals.openedition.org/rfp/1175.

DAUnAY, B. (2013). « Système didactique - Triangle didactique ». In : Reuter, Y. (éd.), Dictionnaire des concepts fondamentaux des didactiques. Louvain-la-Neuve : De Boeck, p. 203-210.

DOLZ, J., JACQUIN, M. \& SCHNEUWLY, B. (2006). « Le curriculum enseigné en classe de français au secondaire : une approche à travers des objets enseignés ». In : Audigier, F., Crahay, M. \& Dolz, J. (éds), Curriculum, enseignement et pilotage. Raisons éducatives. Bruxelles : De Boeck, p. 143-164. DUFAYS, J.-L., GEMENNE, L. \& LEDUR, D. (2005) [1996]. Pour une lecture littéraire. Bruxelles : De Boeck. HALTÉ, J.-F. (1992). La didactique du français. Paris : Presses universitaires de France.

LAURENT, J.-P. (2011). « Quelques enjeux didactiques d'une configuration de la discipline "français". Vers le concept d'ajustement didactique ». In : Daunay, B., Reuter, Y. \& Schneuwly, B. (éds), Les concepts et les méthodes en didactique du français. Namur : Presses universitaires de Namur, p. 61-82.

LEgros, G. \& CANVAT, K. (1997). « Enseigner la poésie moderne ?». Pratiques 93, p. 5-29. En ligne : https://www.persee.fr/doc/prati_0338-2389_1997_num_93_1_1792.

maingueneau, D. (2004). Le Discours littéraire. Paratopie et scène d'énonciation. Paris : A. Colin.

maingueneau, D. (2006). Contre Saint Proust. Ou la fin de la littérature. Paris : Belin.

MURPHY, S. (1990). Le premier Rimbaud ou l'apprentissage de la subversion. Lyon : Éd. du CNRS/Presses universitaires de Lyon.

PLESSEN, J. (1967). Promenade et Poésie. La Haye/Paris : Mouton \& Cie.

RANCIÈRE, J. (2017). Le maître ignorant : cinq leçons sur l'émancipation intellectuelle. Paris : Fayard.

REUTER, Y. \& LAHANIER-REUTER, D. (2007). « L'analyse de la discipline : quelques problèmes pour la recherche en didactique ». In : Falardeau, É. et al. (éds), La didactique du français. Les voies actuelles de la recherche. Québec: Presses de l'université Laval, p. 27-42.

RIFFATERRE, M. (1979). La production du texte. Paris : Seuil.

Rimbaud, A. (2009) [1870]. « Vénus Anadyomène ». In : Rimbaud, A., Euvres complètes. Paris : Gallimard, p. 66 . 
RONVEAUX, C. \& SCHNEUWLY, B. (éds) (2018). Lire des textes réputés littéraires au fil des niveaux scolaires. Berne : P. Lang.

SCHNeuwly, B. (2009). « Le travail enseignant ». In : Schneuwly, B. \& Dolz, J. (éds), Des objets enseignés en classe de français. Rennes : Presses universitaires de Rennes, p. 29-43.

VÉDRINES, B. \& GABATHULER, C. (2018). « De la réputation "littéraire” ». In : Schneuwly, B. \& Ronveaux, C. (éds), Lire des textes réputés littéraires au fil des niveaux scolaires. Berne : P. Lang, p. 67-81.

VUILLET, Y. (2017). À la recherche didactique de concepts pour penser, dire et agir le littéraire. Thèse de doctorat en sciences de l'éducation, Université de Genève.

VUILLET, Y. \& GABATHULER, C. (2017). « Approcher l'enseignement du littéraire comme essentiellement contestable ». In : Brunel, M. et al. (éds), L'enseignement et l'apprentissage de la lecture aux différents niveaux de la scolarité. Namur : Presses universitaires de Namur, p. 51-77.

\section{NOTES}

1. Désormais : V.A.

2. Cette séquence d'enseignement s'est réalisée dans le contexte d'un dispositif de formation à l'enseignement de la littérature. L'enseignant dont les activités seront analysées a collaboré, lors d'une formation préalable, à la construction collective d'une séquence didactique plaçant en son centre la V.A. d'A. Rimbaud. C'est en exploitant et en réorganisant les outils d'enseignement ainsi conçus - mais aussi en leur ajoutant des tâches originales - que l'enseignant a réalisé la séquence d'enseignement retenue pour cet article, avec ses élèves âgés de 13 à 14 ans.

3. Voici l'extrait, et qui synthétise également l'interprétation que S. Murphy (1990, p. 209) propose de la V.A. : « Lorsque Michael Riffaterre prétend que ce poème ne contient "ni polémique morale", ni "controverse esthétique", il ramène le sonnet à une esthétique formaliste, antiidéologique. Chemin faisant, il reproduit en large mesure la problématique esthétique et idéologique du Parnasse, qui est précisément la cible de Vénus Anadyomène [...]. Comme à son habitude, Rimbaud souligne de manière provocatrice les rapports entre morale et esthétique. Il ne s'agit pas d'une esthétique de la laideur, mais d'une représentation non-réaliste de la réalité de la prostitution. Le corps de cette Vénus constitue comme une formule, résumant l'étiologie de la prostitution et son contexte littéraire et artistique. Son caractère réflexif ne saurait cependant déguiser son propos subversif, son message portant sur une réalité extra-linguistique. »

4. Développé par l'équipe genevoise du Groupe de recherche et d'analyse du français enseigné (GRAFE), la macrostructure condense les informations tirées d'une analyse des activités d'enseignement et d'apprentissage effectuée à partir des interactions orales intégralement transcrites. La macrostructure restitue le déploiement d'un objet d'enseignement à travers la succession de tâches et de modules d'enseignement.

5. La figure 1 ne restitue ni les répétitions de modules, ni les interruptions d'un module par un autre qui interviennent parfois.

6. Pour des développements, je me permets de renvoyer à Vuillet, 2017.

7. Les élèves sont répartis en groupes de quatre. Chaque membre d'un groupe se voit attribué soit un quatrain, soit un tercet du sonnet pour le transposer génériquement sous forme de calligramme. Au sein de chaque groupe (six au total), la V.A. est ainsi reconstituée par l'intermédiaire du travail des élèves.

8. Voir notamment Daunay (2013). Pour un exemple d'activation de ce concept au sein d'une analyse didactique d'une situation d'enseignement portant sur un texte réputé littéraire, voir Vuillet \& Gabathuler, 2017. 
9. Ce qu'illustre cet extrait d'interaction scolaire réalisée au cours du module 10 , alors que l'enseignant explicite le sens qu'il met sur la transformation scolaire de la V.A. en calligramme : Ens. : " quelque part: repartir du texte de Rimbaud: et en refaire une peinture: en refaire un dessin: en faire un calligramme: réécrire un autre poème: c'est tout à fait dans la logique des choses: dans la logique de l'avancée de l'histoire de l'art quelque part $\downarrow$ ”

10. Le terme est employé à plusieurs reprises par l'enseignant au cours de la séquence, à l'exemple de cette précision posée dès le premier module: Ens : «mais je reste tout à fait d'accord sur une chose : c'est qu'il peut y avoir un côté récréatif à lire de la poésie/ et dans ce cas-là : on garde la racine du mot: on n'est pas dans le distraire: c'est-à-dire détourner l'attention : mais plutôt dans l'idée de création et de recréer : la récréation ça sert à pouvoir ensuite créer de nouveau/ tu vois ce que je veux dire : " El15 : oui Ens :-récréation- se RECREER. 11. Ce constat ne vise pas à mettre en compétition les légitimités respectives des activités scolaires et des activités d'experts littéraires : il permet, d'une façon plus descriptive, de leur reconnaitre des pertinences équivalentes en fonction de leurs contextes propres. Il suggère encore que la description et la compréhension de ce qu'il se passe en classe lorsque des poèmes font l'objet d'enseignements invite, en fonction du projet, à ne pas nécessairement prendre les activités expertes comme seule référence.

12. Il en va d'ailleurs de la même manière pour tout objet de connaissance.

13. C'est pour approcher ces problèmes dans une perspective didactique que j'ai proposé ailleurs le concept de nœud idéologique (Vuillet, 2017 ; Vuillet \& Gabathuler, 2017).

\section{RÉSUMÉS}

Cet article propose d'approcher la Vénus Anadyomène d'A. Rimbaud dans une perspective comparatiste et didactique. Ce qui a pu être dit et fait de ce poème dans deux contextes distincts, à savoir ceux d'études littéraires et celui d'une classe de français, est analysé de façon à rendre visible la dépendance forte que ces deux ensembles d'activités entretiennent respectivement avec leurs propres contextes de réalisation. Dans la première partie, qui emprunte aux propositions formulées par D. Maingueneau au sujet du discours littéraire, l'article analyse et compare des commentaires produits par trois experts académiques, sur une période d'une vingtaine d'années, au sujet du sonnet retenu. Certaines différences aussi bien que certaines régularités de ces études littéraires sont mises en évidence. Dans la seconde partie, c'est cette fois la transformation de la Vénus Anadyomène en objet d'enseignement, dans le cadre d'une classe du secondaire I genevois, qui se trouve décrite dans une perspective didactique. Des différences notables entre le traitement académique et le traitement scolaire du sonnet d'A. Rimbaud sont soulignées. Finalement, cet article se conclut par une réflexion qui porte sur les différences qui séparent, à l'endroit du travail avec les textes poétiques, les activités des commentateurs académiques experts et celles des enseignants. Les analyses développées suggèrent notamment de reconnaitre l'originalité, et la légitimité du travail scolaire avec des textes poétiques.

This article approaches A. Rimbaud's Venus Anadyomène through a comparative and didactic perspective. The analysis of uses and interpretations of the poem in both literary studies and in a French class context aims to demonstrate how these different types of activities are orientated by their own contexts. In the first part, which follows D. Maingueneau's propositions on literary discourses, the article analyzes and compares comments made by three Rimbaud scholars, over a 
time span of about twenty years. A number of differences as well as similarities of these literary studies are highlighted. In the second part, Venus Anadyomène's transformation into a teaching subject is described from a didactic perspective. Significant differences between the academic treatment and the school treatment of A. Rimbaud's sonnet are underlined. Finally, comparing the specificities of the teacher's work to that of the critic, this article suggests to recognise the originality and the legitimacy of classroom work with poetic texts.

INDEX

Mots-clés : approche didactique de la poésie, analyse de l'activité, séquence didactique, discours littéraire

Keywords : didactic approach of poetry, teaching's activity analysis, didactic sequence, literary discourse

\section{AUTEUR}

\section{YANN VUILLET}

Haute École Pédagogique du Valais, CH-1890, Suisse 